\title{
Prior knowledge does not facilitate the perceptual organization of dynamic random-dot patterns
}

\author{
JOSEPH S. LAPPIN \\ Vanderbilt University, Nashville, Tennessee 37240 \\ and \\ JOSHUA D. STALLER \\ State University of New York, Oswego, New York 13126
}

\begin{abstract}
Three classes of models for the origins of perceptual organization-linear independence, cognitive facilitation, and self-organization-were tested by evaluating the effects of stimulus unccitainty and pattern coherence on the detection of coherent motion in dynamic random-dot patterns. Stimulus patterns consisted of two successive frames of randomly positioned dots in which motion was perceived when the dot positions in successive frames were correlated and displaced. The number of alternative directions and locations of motion and the degree of coherence (correlation) between the two successive frames were manipulated. The effects of stimulus uncertainty were less than predicted by cognitive facilitation models and were less than predicted by one linear independence model (choice theory), although similar to the predictions of another linear model (the Peterson, Birdsall, \& Fox, 1954, approximation of the optimum Gaussian model). Small, but significant, tendencies toward self-organization rather than linear independence of perceived motion in neighboring locations were indicated by a nonlinear effect of coherence on detection accuracy and by the superior detectability of the direction as compared with the location of motion.
\end{abstract}

Visual stimulation often consists of large numbers of more or less separate components discontinuously distributed over space or time, or both. The recognition of such patterns involves the detection of organized relationships among the components. Is the perceptual organization of such patterns facilitated by prior knowledge about the stimulus pattern? The purpose of this study was to answer this question for a particular set of dynamic patterns which produced the perception of motion.

Specifically, observers detected coherent ${ }^{1}$ motion embedded in otherwise random patterns consisting of two successive displays of over 500 randomly positioned spots of light. Prior knowledge of the organization was manipulated by varying the number of alternative directions or locations of motion while the number of response alternatives was held constant. The organization of these patterns was also manipulated by varying the correlation between the positions of dots in successive display frames. The primary experimental question was how the detectability of or-

This research was supported by NSF Research Grant BNS-7805857 and Postdoctoral Fellowship 2-F32-MH05499. We gratefully acknowledge the contributions of several anonymous reviewers in correcting several errors and potential misinterpretations in previous versions of this manuscript. Correspondence may be addressed to Joseph S. Lappin, Department of Psychology, Vanderbilt University, Nashville, Tennessee 37240. ganization would be influenced by the number of alternative directions or locations of motion.

We may distinguish three different classes of models of perceptual organization, one linear and two nonlinear:

(1) Linear independence models. The perception of coherent motion may derive from the operation of a linear sensory system that does not qualitatively transform the visual representation of the physical stimulus patterns. Perceptual organization may correspond to stimulus organization. In the present experiments, perceived motion of the whole stimulus pattern may correspond to a linear sum of the perceived motion of all the individual components; perceptual information about the displacement of any given component may be stochastically independent of information about the displacement of any other component. Accordingly, prior knowledge about the set of potential stimulus patterns would have no influence on the organization of events within such a linear system.

(2) Cognitive facilitation models. The sensory information arising from complex stimulus patterns is sometimes considered as an unorganized "buzzing confusion" in which order is imposed by cognitive recoding in accord with previous experience and knowledge. Contemporary models of perception and cognition often postulate that internal representa- 
tions of stimulus patterns are constructed by a combination of both stimulus-based and knowledgebased processes. Selective attention to a particular stimulus organization may enhance the development of a corresponding perceptual representation. Thus, prior knowledge of the direction or location of motion may improve the detection of coherent motion.

(3) Self-organizing models. As suggested by traditional Gestalt ideas, perceptual processes may be selforganizing, controlled by cooperative visual interactions among components of the stimulus pattern rather than by knowledge of alternative organizations. An extreme form of such a system exhibits an organizational threshold with only two alternative states. If visual perception operated as such a threshold process, detection performance would be unaffected by stimulus uncertainty. In general, however, self-organizing processes are likely to produce less abrupt transitions in state, yielding some small effects of stimulus uncertainty on performance.

Experimental evidence can be found for each of these classes of models. Before considering this evidence, however, let us examine the rationale that associates these perceptual models with observed detection performance.

Even under a linear independence model in which the observer's prior knowledge of the stimulus has no effect on perceptual organization, detection accuracy should decrease with a larger number of stimulus alternatives. A linear model constitutes a form of null hypothesis, or normative model, against which the experimental results must be evaluated. Specific quantitative predictions cannot be given for the other two nonlinear models, so they must be evaluated by reference to the normative predictions of a linear independence model. Thus, the cognitive facilitation models predict a greater effect of the number of stimulus alternatives on detection accuracy, whereas selforganizing models predict a smaller effect than the linear models.

Detection accuracy should be expected to decrease with a larger number of stimulus alternatives, because the information associated with a given physical event depends upon the set of alternatives, even when the response alternatives are held constant. With more stimulus alternatives in a given response category, more physical parameters are relevant and the likelihood ratio for any given physical event is smaller. Although the sensory representation of a given physical stimulus event may be independent of the set of alternatives, the information it provides is diluted by the addition of sensory information about other potential stimulus alternatives; the signal/noise ratio is lowered by the addition of noise from other sensory channels. Even a theoretically ideal detection system cannot avoid this limitation imposed by uncertainty about the physical parameters of the stimulus. Thus, changes in detection performance pro- duced by changes in the size of the stimulus set may have less to do with perceptual processes than with the stimulus information that is processed. ${ }^{2}$ Several authors have shown how such linear independence models provide a quantitative account of many of the experimental effects of stimulus uncertainty without invoking the intervention of special perceptual mechanisms (e.g., Cohn \& Lasley, 1974; Green \& Birdsall, 1978; Kinchla, 1974, 1977; Lappin, 1978; Lappin \& Uttal, 1976).

A basic assumption in most linear models is that the stimulus alternatives are orthogonal in the sense that their distinguishing features are uncorrelated. "Noise" that perturbs the detection of any given stimulus is assumed to be independent of the noise that perturbs any other stimulus. Thus, if there are $n$ stimulus alternatives, there may be $n-1$ independent opportunities for the noise to be mistaken for the true target stimulus in any given presentation.

A number of different linear models can be constructed, depending upon assumptions about the distribution of hypothetical noise and assumptions about the decision rule for selecting a response to perceptual information about multiple stimulus alternatives. In evaluating the present data, we employed two different linear models based on two different decision rules. While other plausible models can be constructed, these two provide perspective on the range of predictions that might be obtained. ${ }^{3}$

One model was an approximation formulated by Peterson, Birdsall, and Fox (1954) for the optimal Bayesian decision rule. The optimal decision rule involves summing the likelihood ratios over all of the stimulus alternatives within each response category and then selecting the response associated with the highest total likelihood ratio. When the underlying noise is normally distributed, the corresponding distribution of likelihood ratios for the composite response category is the distribution of a sum of independent log-normally distributed random variables. The Peterson et al. approximation assumes that the distribution of the log likelihood ratio is normal (as it is when there is only one stimulus alternative). The resulting formula is

$$
\mathrm{d}_{\mathrm{n}}^{\prime}=\left\{\ln \left[\frac{\exp \left(\mathrm{d}_{1}^{\prime}\right)+\mathrm{n}-1}{\mathrm{n}}\right]\right\}^{1 / 2},
$$

where $d_{n}^{\prime}$ and $d_{1}^{\prime}$ are the detection measures for conditions with $\mathrm{n}$ and with one stimulus alternative, respectively. While the Peterson et al. approximation is quite good at lower performance levels, say $\mathrm{d}^{\prime}<1.0$, it is known to erroneously predict better performance than the optimal model at higher performance levels (see Nolte \& Jaarsma, 1967). In the present experiments, however, the overestimation (assuming normally distributed noise) is statistically significant only at the higher performance levels in one condition (the 
four-alternative forced-choice task with four stimulus alternatives). Accordingly, we regard these predictions as indicative of an upper bound on the performance to be expected for an independence model.

A second linear independence model was based on Luce's choice theory (Luce, 1959, 1963; Shipley, 1960). An important characteristic of this specific model is the decision rule, which is to select the response associated with the stimulus alternative with maximum amplitude or likelihood ratio. Not only is this a psychologically plausible decision procedure, but the model is analytically simple. Assuming that all of the stimulus alternatives have an independent and equal opportunity of being mistaken for the true target stimulus (having a higher likelihood ratio), it is easy to compute the decreasing probability that the true target will emerge as the maximum when the number of alternatives is increased. The effect of the number of stimulus alternatives, $n$, can be expressed in a form similar to Equation 1 above:

$$
D_{n}=(1 / \sqrt{2}) \ln \left[\frac{\exp \left(\sqrt{2} D_{1}\right)+n-1}{n}\right] \text {, }
$$

where $D_{n}$ and $D_{1}$ are the detection measures, $-\log \eta$, for conditions with $\mathrm{n}$ and with one stimulus alternative (see Appendix; Lappin \& Uttal, 1976).

Compared with the Peterson et al. (1954) model, the choice-theory model yields a greater drop in performance from increasing the number of stimulus alternatives. The discrepancy between the predictions of these two models increases with the number of alternatives and with the detectability of the target stimulus.

Lappin and Uttal (1976) found that the choicetheory model provided a good description for the decreasing accuracy of detecting a target stimulus camouflaged in a background of random dots as the number of alternative locations was increased from two to eight possible locations. Similarly, this model also provides a good description for the results of Cohn and Lasley (1974) on the effect of spatial uncertainty on the detectability of incremental light flashes. The results of both experiments were closer to the predictions of the choice-theory model than to those of the Peterson et al. (1954) model. (We will report more details of this comparison in a later section.) Thus, the results of both experiments were consistent with a linear model, indicating no attentional influence on perceptual organization.

In contrast with the preceding results, a few experiments have suggested the influence of a cognitive or attentional process that facilitates detection when stimulus parameters are known beforehand. An especially relevant study of motion detection was conducted by Sekuler and Ball (1977). Observers in their experiment were less accurate in detecting motion (continuous motion of randomly positioned dots) in one of two alternative directions than in detecting motion in a single direction that was constant over a block of trials. The difference in accuracy for these two conditions was significantly greater than predicted by either of the linear independence models. Apparently, prior knowledge of the direction of motion did facilitate detection in that situation. Shaw (in press) also has recently reported results of experiments on tachistoscopic letter detection in which the effects of uncertainty about spatial location were greater than would be attributed to a linear independence model. Whether and how such cognitive processes might be involved in the perceptual organization of coherent motion in random-dot patterns remains uncertain, however.

Indeed, several studies of the perception of textured patterns of large numbers of random elements suggest self-organizing processes. Apparently, the perceptual organization of such patterns may often be determined "spontaneously" and "automatically" by processes involving cooperative perceptual interactions among the stimulus components, prior to any cognitive analysis by the observer (e.g., Beck, 1967; Julesz, 1975, 1978; Pollack, 1973; Staller, Lappin, \& Fox, 1980). A single organization may dominate perception so that the observer obtains little information about any alternative organization of the pattern. Staller et al. (1980) recently found that the stereoscopic organization of dynamic random-element stereograms was unaffected by the number of alternative target forms. Julesz and Burt (1979) also recently reported that the detection of redundant target forms in a texture-discrimination task was better than expected from independent detections when the component forms were familiar to the observer and grouped in neighboring areas of the pattern, indicating an automatic figure-ground organization of the pattern. In a study of apparent motion, Beck, Elsner, and Silverstein (1977) found that uncertainty about spatial locations did not affect the subjective quality of motion, although the generalization of this result to the detection of coherent motion in complex patterns is uncertain.

The general strategy of the present experiment was to compare the effects of stimulus uncertainty on detection performance with normative predictions derived from linear independence models (choice theory and Peterson et al., 1954). If the effect of stimulus uncertainty was less than predicted, then perceptual processes must be self-organizing; if the effect was greater than predicted, then cognitive facilitation is implicated. We found, in general, that the effect of uncertainty was less than predicted by the choicetheory model but similar to the predictions of the Peterson et al. model-rejecting cognitive facilitation models but equivocal with regard to linear independence models. Additional analyses and tests of the interdependence of perceived motions in neigh- 
boring spatial locations indicated that self-organizing processes contributed to the detection of coherent motion in these stimulus patterns.

\section{METHOD}

\section{Subjects}

The authors and three paid Vanderbilt student volunteers were tested individually. All were well-practiced on this type of task.

\section{Stimuli}

The stimulus patterns were pairs of successively presented, spatially superimposed, random-dot displays. When the positions of dots in successive display frames were correlated and displaced horizontally or vertically, the pattern was seen to move. Each irame consisted of 512 dots positioned randomly in the cells of a 32 by 32 matrix, with the constraint that each row and each column contain 16 dots. Each pattern consisted of two display frames presented in succession. The spatial and statistical relationship between the two frames of a pair was systematically varied. The dots in the second frame could be horizontally or vertically displaced one row or column with respect to the dots in the first frame. In addition, the proportion of correlated and displaced elements could be specified. For instance, all the dots and empty cells in the second frame could be displaced one row or column with respect to the first frame (a proportion of 1.0); or half the elements could be displaced, with the remainder randomly regenerated (a proportion of 0.5 ). A proportion of 0.0 meant that the positions of the dot elements in both frames were independently randomly generated. The computer algorithm permitted selection of 1 of 17 different proportion levels between (and including) 0.0 and 1.0 . It is important to note that the outside boundaries of both of the two successive frames were always in the same spatial position. Thus, when the pattern was displaced to the right, for example, the column of dots at the far right edge of the first frame was removed and reinserted at the left edge of the second frame (the identity of these two subsets was not detectable).

For several of the experiments reported here, the 32 by 32 pattern was partitioned by computer algorithm into four adjacent, but nonoverlapping, 16 by 16 patterns. One of the 16 by 16 quadrants contained a correlated displacement, while the other three quadrants contained random sequences. The location of the correlated quadrant, the direction of displacement, and the proportion of correlated cells were all under computer control.

The computer-generated dot patterns were displayed on a cathode ray tube (Tektronix, 604) with rapidly decaying phosphor (P-15). The dots in each frame were continually replotted at a rate of $19.5 \mu \mathrm{sec} /$ dot (approximately $10 \mathrm{msec}$ for the complete set of 512 dots) until the desired exposure duration was reached (200 $\mathrm{msec} /$ frame with no interframe interval). The 32 by 32 matrix subtended approximately $3 \times 3$ deg of visual angle. All patterns were binocularly viewed in a dimly lit room from a distance of $86 \mathrm{~cm}$. The observers' heads were positioned by a chinrest.

\section{Procedure}

Temporal forced-choice. One part of the experiment involved a two-alternative temporal forced-choice detection task in which two separate patterns were presented on each trial. In one pattern, a proportion of the ( 32 by 32 ) cells in the two successive frames were identical, but horizontally or vertically displaced by one row or column (the displaced cells were distributed randomly throughout the pattern), and the remainder of the cells were generated randomly. In the other pattern, the two successive frames were uncorrelated. The two patterns of a given trial were separated by $.5 \mathrm{sec}$.

The observer's task was to identify which of the two patterns was coherently moved. The main experimental manipulation concerned the number of alternative directions in which the pattern could be moved within a given block of trials. One set of test ses- sions compared the detectability of one vs. two directions of motion (left, right). A second set of test sessions compared one vs. four directions of motion (left, right, up, down).

Additionally, detectability was varied by manipulating the proportion of correlated cells in the target pattern. Proportions of $.0605, .121, .182$, and .242 were used $(1 / 16,2 / 16,3 / 16$, or $4 / 16$ $\times 31 / 32$ ). The observers initiated a trial by depressing two telegraph keys aligned side by side. Responses were signaled by lifting one of the keys-the left key corresponded to the first display interval and the right key to the second interval. A fixation point was continuously displayed except during stimulus presentation.

Spatial forced-choice. A four-alternative spatial forced-choice task extended the investigation to other stimulus and response conditions. The 32 by 32 matrix was partitioned into four 16 by 16 quadrants. A single pair of successive frames was presented on each trial, and the coherent motion occurred in only one of the four quadrants. In the other three quadrants, the successive frames were uncorrelated.

There were two variations of the spatial forced-choice proce. dure. In one variation, observers identified the location of the quadrant that contained coherent motion. The direction of motion was either consistent within a trial block or it occurred with equal probability in any one of four directions (left, right, up, down). Responses were signaled using three telegraph keys. One key was used to initiate trials and record responses. Two other keys provided a binary code by which observers could indicate the quadrant that contained the coherent motion.

In a second variation, observers were asked to identify the direction of motion. There were four alternative directions, and in separate experimental conditions the number of alternative locations of the coherent motion was varied. In one condition there were two locations-in either of two diagonally related quadrants. The diagonal was consistent throughout a trial block and counterbalanced across trial blocks. In the other condition, the motion occurred in any one of the four quadrants. The patterns in this latter condition were identical to those in the spatial forced-choice version in which observers identified the location of the coherent motion in one of four directions; only the stimulus-response mapping was changed. In both spatial forced-choice tasks, discriminability was varied by manipulating the degree of correlation in the target quadrant $(.176, .352, .527$, or .703 ; equal to $3 / 16,6 / 16$, $9 / 16$, or $12 / 16 \times 15 / 16)$.

General. In both temporal and spatial forced-choice conditions, feedback about accuracy was provided on each trial by a Teletype bell, and the intertrial interval was self-paced. In general, each manipulation (temporal, I vs. 2 vs. 4 directions; spatial, identify location and identify direction) required four daily 1 -h test sessions per subject. Within a session there were four blocks of trials, 100 trials per block, with a short break between blocks. The number of stimulus alternatives was varied within a session; for instance, observers would receive 100 trials in which the direction of motion was constant, followed by 100 trials in which the direction was randomly varied. There were 200 trials for each observer under each experimental condition. Within a session, the degree of coherence of the target pattern was held constant, and the order in which observers received the four different coherence levels was counterbalanced as much as possible.

\section{RESULTS}

The results of these experiments are shown in Figures 1 and 2. For each experimental task, the percentages of correct detections in two conditions differing in the number of stimulus alternatives are illustrated along with the predictions of two linear independence models-the choice-theory model and the Peterson et al. (1954) approximation of the optimal model. The averaged predictions shown in Figures 1 and 2 were for 


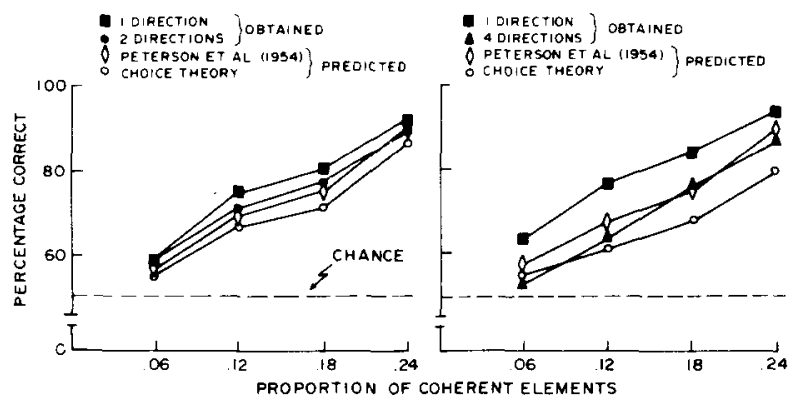

Figure 1. Average detection accuracies in the two-alternative temporal forced-choice task. Left panel: Obtained data for one and two alternative directions of motion, and average predictions of the Peterson, Birdsall, and Fox (1954) approximation of the optimal model and of the choice-theory model for the two-direction condition. Right panel: Obtained data for one and four alternative directions of motion. Predictions of the Peterson et al. and choice-theory models were based on the performance of each observer in the one-direction condition.

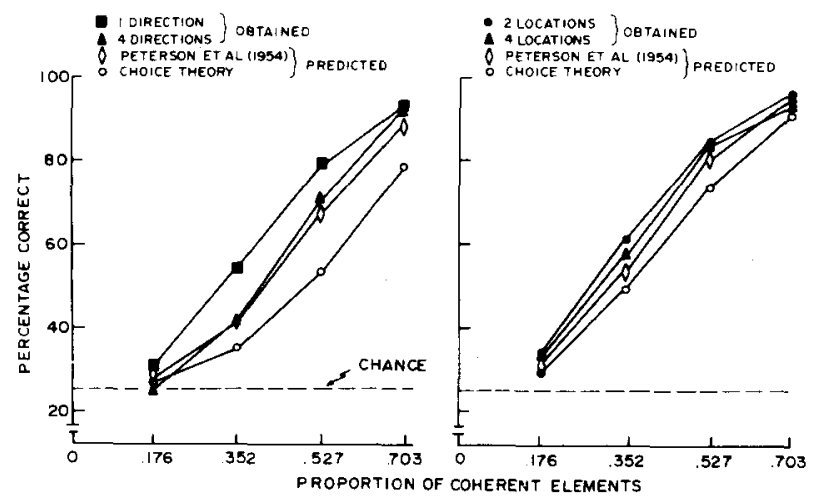

Figure 2. Average detection accuracies in the four-alternative spatial forced-choice tasks. Left panel: Observers identified the location of motion in either one or four alternative directions. Right panel: Observers identified the direction of motion in either two or four alternative locations.

performance in the condition with more alternatives as derived from the performance with fewer alternatives at the same stimulus coherence level. (The choicetheory model is derived in the Appendix.)

As may be seen, detection accuracy was consistently lower when the number of stimulus alternatives was increased. This occurred in 46 of $52 \mathrm{com}$ parisons of the data of individual observers, with the few exceptions scattered unsystematically over the experimental conditions. The obtained performance was close to the predictions of the Peterson et al. model at all levels of accuracy. In contrast, the discrepancy between the obtained performance and the predictions of the choice-theory model tends to increase with the accuracy of detection. These findings appear rather consistent across the several different experimental tasks.
Statistical analyses used the binomial distribution to measure differences between obtained and predicted percentages of correct responses. Specifically, the chi-square measure of deviation is $\chi^{2}=\mathrm{N}(\mathrm{O}-\mathrm{P})^{2}$ / $P(1-P)$, where $O$ and $P$ are the obtained and predicted percentages of correct responses, and $\mathrm{N}$ is the number of trials ( $\mathrm{N}=\mathbf{2 0 0}$ in the present experiments). Each of the normative linear models (choice theory and Peterson et al.) was fit to the data from the two uncertainty conditions for each observer under each coherence level in each experimental task. An adaptive search program was used to find the detection parameter that minimized the chi-square measure of fit of the model to the data for the two different uncertainty conditions. Thus, there is 1 degree of freedom for fitting each of the one-parameter models to the data of the two conditions. Table 1 gives the resulting chi-square measures summed over observers at each coherence level in each task. The degrees of freedom for each entry are equal to the number of observers in that condition.

These statistics generally corroborate the impressions provided by Figures 1 and 2 . The magnitude of the effects of uncertainty predicted by the choicetheory model typically were significantly greater than the obtained effects. The few exceptions to this trend occurred at the lower levels of pattern coherence, at which detection was less accurate. The Peterson et al. predictions, on the other hand, were typically close to the obtained performance. The most noticeable exception to the latter relationship occurred in the four-direction condition of the temporal forcedchoice task, in which the obtained difference between the two conditions was significantly greater than the predicted values, although in most other conditions the deviation was in the opposite direction. Over the total of 52 comparisons for each observer and experimental condition, the Peterson et al. model predicted a larger effect than was obtained in 31 cases (not significantly different from the expected value of 26 ). It should be remembered that the Peterson et al. model slightly underestimates the effect of uncertainty on a theoretically optimal decision-maker (assuming normally distributed noise) at higher levels of detectability (Nolte \& Jaarsma, 1967).

The above results do not permit a rejection of the general class of linear independence models. Two additional pieces of evidence are relevant, however. First, consider the effect of the number of coherent elements on the accuracy of detecting the coherent motion. If each of the component elements was perceptually independent, then its contribution to the overall percept ought to be linearly independent or additive. Accordingly, detection accuracy, as measured by $\mathrm{d}^{\prime}$ or the choice-theory measure $-\log \eta$, should be directly proportional to the proportion of coherent elements. ${ }^{4}$ 
Table 1

Chi-Square Measures of Fit of Each of Two Models in Each Experimental Condition

\begin{tabular}{|c|c|c|c|c|c|c|c|c|c|c|c|c|c|}
\hline \multicolumn{6}{|c|}{ Two-Alternative Temporal Forced Choice } & \multicolumn{6}{|c|}{ Four-Alternative Forced Choice } & & \\
\hline \multicolumn{3}{|c|}{$\begin{array}{l}\text { One vs. Two } \\
\text { Directions (2) }\end{array}$} & \multicolumn{3}{|c|}{$\begin{array}{l}\text { One vs. Four } \\
\text { Directions }(6)\end{array}$} & \multicolumn{3}{|c|}{$\begin{array}{l}\text { Spatial: One vs. Four } \\
\text { Directions ( } 2 \text { ) }\end{array}$} & \multicolumn{3}{|c|}{$\begin{array}{l}\text { Directional: Two vs. Four } \\
\text { Locations (3) }\end{array}$} & \multicolumn{2}{|c|}{$\begin{array}{c}\text { Grand } \\
\text { Total }(52)\end{array}$} \\
\hline $\mathrm{C}$ & $\mathrm{CT}$ & $\mathbf{P}$ & $\mathrm{C}$ & $\mathrm{CT}$ & $\mathbf{P}$ & C & CT & $\mathbf{P}$ & $\mathrm{C}$ & CT & $\mathbf{P}$ & $\mathrm{CT}$ & $\mathbf{P}$ \\
\hline $\begin{array}{l}.06 \\
.12 \\
.18 \\
.24\end{array}$ & $\begin{array}{l}2.51 \\
2.39 \\
4.36 \\
3.41\end{array}$ & $\begin{array}{r}1.27 \\
.29 \\
1.02 \\
1.19\end{array}$ & $\begin{array}{l}.06 \\
.12 \\
.18 \\
.24\end{array}$ & $\begin{array}{c}3.18 \\
9.75 \\
31.16^{*} \\
33.30^{*}\end{array}$ & $\begin{array}{c}9.68 \\
11.29 \\
5.45 \\
17.24 * *\end{array}$ & $\begin{array}{l}.18 \\
.35 \\
.53 \\
.70\end{array}$ & $\begin{array}{c}.06 \\
5.02 \\
22.21^{*} \\
22.97^{*}\end{array}$ & $\begin{array}{l}.48 \\
.81 \\
.57 \\
3.70\end{array}$ & $\begin{array}{l}.18 \\
.35 \\
.53 \\
.70\end{array}$ & $\begin{array}{c}5.01 \\
11.31 \dagger \\
21.92^{*} \\
2.32\end{array}$ & $\begin{array}{l}2.72 \\
2.22 \\
9.96 \dagger \\
.34\end{array}$ & & \\
\hline Total (8) & 12.66 & 3.77 & Total (24) & $77.39 *$ & $43.66 * *$ & Total (8) & $50.26^{*}$ & 5.57 & Total (12) & $40.55^{*}$ & 15.24 & $180.86^{*}$ & 68.24 \\
\hline
\end{tabular}

Note $-C=$ coherence; $C T=$ choice theory, $P=$ Peterson et al. (1954). Numbers in parentheses are degrees of freedom. ${ }^{*} p<.001 . \quad * * p<.01 . \quad t_{p}<.05$.

This hypothesis was tested by examining the choicetheory measure, $-\log \eta$, in the conditions with only one alternative direction of motion. (In the conditions with more stimulus alternatives, the prediction would depend on additional assumptions about the effect of the uncertainty.) Graphically, the question was whether a plot of $-\log \eta$ vs. proportion of coherent elements was a straight line through the origin of the graph. Thus, one free parameter was used to describe four data points (one for each coherence level) for each observer in each appropriate condition. The goodness of fit of the obtained data to this theoretical function was assessed by the chi-square measure.

For each of the three experimental conditions in which the motion occurred in only one direction, the obtained data deviated significantly, although slightly, from the predicted function. While the obtained data were usually close to a straight line, the intercept of this line on the horizontal axis was usually at a point above the zero coherence level, indicating a threshold in detecting low levels of coherence.

In the temporal forced-choice task comparing one vs. two directions, the data for neither of the two observers were significantly different from the predicted function, but the combined chi-square for both observers was statistically significant $\left(\chi^{2}=13.24\right.$, $\mathrm{df}=6, \mathrm{p}<.05$ ). In the temporal forced choice task comparing one vs. four directions of motion, the data for three of the six observers ${ }^{5}$ deviated significantly $(\mathrm{p}<.05)$ from the predicted values, and the combined chi-square for all six was statistically significant $\left(\alpha^{2}=39.36, \mathrm{df}=18, \mathrm{p}<.01\right)$. In the spatial forced-choice task comparing one vs. four directions of motion, the data for both observers were significantly $(\mathrm{p}<.001)$ different from the predicted values, and of course the combined chi-square was also statistically significant $\left(\chi^{2}=63.52, \mathrm{df}=6, \mathrm{p}<.001\right)$. Similarly, large departures were also observed in the directional forced-choice task comparing two vs. four locations of motion, although there is some question about whether the theoretical function should be a straight line in this case with two alternative locations.

A second piece of evidence against the linear independence models derives from comparison of the spatial and directional forced-choice tasks. When four stimulus alternatives were associated with each of the four response alternatives, both tasks involved the same set of 16 stimulus patterns and differed only with respect to the stimulus-response mapping. By the linear independence models, performance of the two tasks should be equal. As may be seen in Figure 2, however, identifications of the direction of motion were more accurate than identifications of the location of motion (in seven of eight comparisons for the two observers who participated in both tasks). Subsequent to the principal experiments, we conducted a more direct comparison of these two tasks, using the same stimulus patterns and varying only the stimulus-response mapping. Three observers each performed 300 experimental trials under each of the two conditions at a single coherence level (.35).

For each of the three observers, accuracy was significantly higher in the directional forced-choice task requiring identification of the direction of motion occurring at one of four locations. The average accuracy was $55 \%$ in the directional forced-choice task and $43 \%$ in the spatial forced-choice task. The total chi-square summed over all three observers was $\mathbf{2 8 . 1 0}$ $(\mathrm{df}=3, \mathrm{p}<.001)$. Thus, discriminations between directions of motion were easier than discriminations between locations of motion of the same patternsapparently because coherent motion in one location tended to induce the perception of corresponding motion in neighboring locations. This induced correspondence in the perceived motion in separate location indicates the operation of a self-organizing process.

\section{DISCUSSION}

The general problem addressed by these experiments concerns the relative influence of present stim- 
ulation and prior knowledge on the perceptual organization of visual patterns. Contrary to the predictions of cognitive facilitation models, the finding of these experiments was that prior knowledge of the direction or location of motion did not facilitate perceptual organization in these dynamic random-dot patterns. Although detection performance was affected by stimulus uncertainty, the magnitude of the effect was less than would be predicted if the perceptual representation were itself modified by cognitive or attentional processes. Indeed, supplementary evidence about the interdependence of perceived motion in neighboring spatial locations suggested the influence of nonlinear self-organizing processes, associated with the intrinsic geometry of the stimulus pattern rather than the structural characteristics of the perceptual system. Nevertheless, the departures were small from the performance of a linear system in which the representation of any component stimulus event was independent of neighboring events and of prior knowledge about the overall pattern.

Since the empirical result was that stimulus uncertainty did affect detection accuracy, how can we rule out the contribution of prior knowledge on perception? Contemporary researchers hold diverse opinions about this question. Behavioral evidence per se is equivocal about the extent to which the effects arise in perceptual processes or in the selection of responses. However, the words of Green and Swets (1966) are usefully recalled:

If a change in the stimulus situation makes the signal harder to detect for the ideal observer, then we shall not be surprised if the same change makes the signal harder to detect for the human observer. The principle is parsimony-it is unnecessary to invent psychological mechanisms to explain a change that may be traced to the stimulus itself (p. 152).

It is unnecessary to invoke attentional or cognitive mechanisms to account for the present effects of prior knowledge on detection.

The comparatively small effect of stimulus uncertainty in the present experiments was inconsistent with cognitive facilitation models, but the magnitude of this uncertainty effect was insufficient to distinguish between linear independence vs. self-organizing models of perceptual organization. The results were clearly inconsistent with one linear model-the choicetheory model-but were compatible with another model-the Peterson, Birdsall, and Fox (1954) model. The complication in empirically testing this problem lies in the diversity of potential linear independence models, differing with regard to the stochastic distribution of perceptual events produced by given stimulus conditions and with regard to the observer's decision rule for selecting responses to given perceptual information. Conclusions about perceptual pro- cesses are contingent on assumptions about response processes. Additional experimental evidence is generally required to determine the perceptual processes that underlie observed effects of uncertainty on performance.

To place the present results in better perspective, we compare them with results of other experiments on stimulus uncertainty. The effects of uncertainty in the present experiments were smaller than in some experiments, but were similar to or larger than those in other experiments. Apparently, the determinants of perceptual organization depend upon the specific details of the physical stimulus patterns.

Cohn and Lasley (1974) reported that the effect of spatial uncertainty on detecting light flashes at one vs. four locations was consistent with the prediction of the Peterson et al. model. Their results were closer, however, to the predictions of the choice-theory model, falling between the predictions of the two models. Combined over three observers, the total chi-square deviation from the Peterson et al. prediction was $\chi^{2}=6.34(\mathrm{df}=3, .10>\mathrm{p}>.05)$, whereas the total deviation from the choice theory prediction was $\chi^{2}=2.00(\mathrm{df}=3)$.

The effects of spatial uncertainty on detecting camouflaged forms in the experiments of Lappin and Uttal (1976) were significantly different from the predictions of both the choice-theory and Peterson et al. models, the obtained performance again falling between the predictions of the two models. Summed over the results of four observers in three conditions (with 2, 4, and 8 alternative locations), the deviation from the Peterson et al. prediction was $\chi^{2}=45.42$ $(\mathrm{df}=8, \mathrm{p}<.001)$, and the deviation from the choicetheory prediction was $\chi^{2}=17.61(\mathrm{df}=8, \mathrm{p}<.05)$.

The study of Eriksen and Spencer (1969) employed a different experimental paradigm, but the results may be interpreted by reference to essentially the same linear independence models, as suggested by Eriksen and Spencer and by Kinchla (1974). Their experimental task demanded the detection of a target letter presented at random positions within rapid sequences of $1,3,5$, or 9 letters (Ts and Us plus a single occurrence of the target letter, A, on half of the trials). Consistent with the hypothesis that perceptual information is not transformed by selective cognitive processes, Eriksen and Spencer found essentially no effect from the presentation rate of the sequence or from the position of the target within the sequence. Correspondingly, the systematic and robust effects from the number of displayed letters may be attributed to the statistical characteristics of the stimulus rather than the processing characteristics of the observer. Based on the average data for four observers (Table 1, p. 8), the deviation from the Peterson et al. model was $\chi^{2}=25.73(\mathrm{df}=3, \mathrm{p}<.001)$ and the deviation from the choice-theory model was $\chi^{2}=$ 
103.26 (df $=3, p<.001)$. However, despite the statistically significant deviation (based on 2,880 trials, where a standard deviation corresponds to a difference in hit rates of less than 1\%), the obtained results were quite close to the Peterson et al. predictions. ${ }^{6}$ Thus, the effect of stimulus uncertainty was somewhat less in Eriksen and Spencer's experiment than in the Cohn and Lasley (1974) or Lappin and Uttal (1976) experiments, but similar to that in the present experiments.

Even smaller effects of uncertainty were obtained very recently by Green and Weber (1980) in an experiment on the detection of temporally uncertain auditory signals. Temporal uncertainty was manipulated by varying the number of alternative intervals $(1,5$, or 10) in a rapid sequence of 10 noise bursts (each $50 \mathrm{msec}$ and separated by $100 \mathrm{msec}$ ) in which a pure tone signal might be presented. As Green and Weber showed, the very small effects of temporal uncertainty that occurred in their experiments indicated that detection was governed by an almost all-or-none threshold process. The obtained effects of uncertainty were much less than those predicted by either the choice-theory or the Peterson et al. equations. As reported in Green and Weber's Table I (p. 1307), the overall chi-square fit of the choice-theory model, summed over three signal intensities for each of three observers, was $\chi^{2}=345.07(\mathrm{df}=18, \mathrm{p}<.001)$; the corresponding fit of the Peterson et al. model was $\chi^{2}=$ $42.53(\mathrm{df}=18, \mathrm{p}<.001)$. By comparison, two versions of a high threshold model yielded fits of $\chi^{2}=$ 6.37 and $7.60(\mathrm{df}=9)$. The specific interpretation for these results is presently unresolved. Although the small uncertainty effects might be taken as evidence of a self-organizing threshold process in the auditory system, another interpretation is that the observers always utilized comparative information about all 10 of the temporal intervals, regardless of the potential locations of the signal. Perhaps the rapid sequential presentation of multiple stimulus events is suited to the operation of slower judgmental processes that integrate information distributed over a range of temporally distinct intervals.

One implication of this brief comparison of experimental methods and results is that the functional relation between stimulus uncertainty and detection accuracy depends upon several characteristics of the stimulus and task conditions, presumably reflecting the influence of the organization and stochastic distribution of visual information as well as the decision procedure for responding to this information. Uncertainty effects that are smaller than predicted by linear independence models suggest the operation of self-organizing or threshold-like visual processes, but the source of such nonlinearities cannot be determined from the behavioral effects of uncertainty alone. Threshold-like effects on behavior might arise, for example, from the subject's blinking on some trials and attentively observing on others, from discontinuous response characteristics of peripheral sensory mechanisms, from self-organizing perceptual representations of complex stimulus patterns, or from certain judgmental processes. In the present experiments, a slight, but significant, tendency toward self-organizing perceptual processes is indicated by the effect of coherence on detection accuracy, particularly with the spatial forced-choice stimulus displays, and by the superiority of the detection of direction vs. location of motion. Both of these findings are contrary to the suggestion of Lappin and Bell (1976) that the perceptual organization of these dynamic patterns reflects only the operation of a linear visual process.

If self-organizing, cooperative processes are involved in the perception of these stimulus patterns, the underlying mechanisms are not well understood. The functional importance of self-organizing processes in visual perception, however, has been clearly demonstrated. The most informative program of research has been that of Julesz (e.g., 1971, 1975, 1978; Caelli \& Julesz, 1978; Julesz \& Burt, 1979). The spontaneous figure/ground organization of textured patterns seems to involve a self-organizing process of grouping and segregating similar neighboring elements. Even though individual elements seem to be distinguished primarily by linear transformations involving second-order or dipole statistics, the neighboring elements are not perceptually independent. Nonlinear cooperative interactions among neighboring elements have also been documented in global stereopsis (Julesz, 1971, 1978). Staller, Lappin, and Fox (1980) recently found that stimulus uncertainty had no effect on the speed of resolution of stereoscopic forms in random-element stereograms, indicating that stereoscopic resolution is completed prior to recognition of the target form. Another example of such cooperative interactions in perceptual organization recently observed in our laboratory (Lappin, Doner, \& Kottas, 1980) occurred in the detection of three-dimensional organization of two successive displays. When the displays were fully correlated, the three-dimensional organization was more accurately detected than when the correspondence of dot positions in the two displays was perturbed even slightly, indicating that the three-dimensional organization was globally perceived and more stable than other slightly perturbed patterns. The emergence of a stable perceptual organization in these phenomena occurs automatically, prior to cognitive analysis by the observers.

It should not be assumed that the absence of effects of prior knowledge on the perceptual organization of the present patterns derived from their motion. The results of Sekuler and Ball (1977) clearly 
contradict such a hypothesis. They obtained average accuracies of about .78 and .56 (two-alternative temporal forced-choice) for detecting motion in one and in two alternative directions, respectively. The accuracies in the two-direction condition predicted by the Peterson et al. and choice-theory models would have been about .73 and .69 , both of which are well above the obtained accuracy.

The following procedural differences between their experiment and ours may be noted: (1) Detection accuracy in their experiment was limited by low contrast of the moving dots against a luminous background, whereas the detection of organized motion in our patterns was limited by the low correlation between successive displays of dots with high contrast. (2) The dot patterns in their experiment were continuously moving, whereas the dot patterns in our experiment were presented in two discrete displays with sharp temporal transients. (3) The retinal area subtended by their stimulus patterns was $9 \mathrm{deg}$ - some of their observers commented that movement was more frequently seen in the peripheral areas than in the foveal area-whereas our patterns subtended $3 \mathrm{deg}$. Apparently, the detection of motion relative to the stationary background in the stimulus patterns of Sekuler and Ball was facilitated by prior knowledge of the direction of motion, in contrast with the more spontaneous perceptual organization of our patterns. The explanation for this difference in results is not yet clear.

In conclusion, the contribution of prior knowledge to perceptual organization appears to depend upon the strength of space-time relationships among components of the stimulus pattern. When the stimulus pattern is well-defined by physical relationships among neighboring components in space-time, as measured perhaps by the autocorrelation spectrum (Julesz, 1978; Lappin \& Bell, 1976; Uttal, 1975), then a stable perceptual organization may emerge automatically, without guidance from the observer's attentional processes. In contrast, when the stimulus pattern consists of physically unrelated components, such as letters in printed text, then the observer's familiarity with a culturally defined pattern of relationships may significantly facilitate the recognition of that pattern (Staller \& Lappin, 1979, in press).

\section{REFERENCE NOTE}

1. Green, D. M. Personal communication, 1977.

\section{REFERENCES}

BEck, J. Perceptual grouping produced by line figures. Perception \& Psychophysics, 1967, 2, 491-495.

Beck, J., Elsner, A., \& Silverstein, C. Position uncertainty and the perception of apparent movement. Perception \& Psychophysics, 1977, 21, 33-78.

Caelli, T., \& Julesz, B. On perceptual analyzers underlying visual texture discrimination: Part I. Biological Cybernetics, $1978,28,167-175$.

COHN, T., \& LASLEY, D. Detectability of a luminance increment: Effect of spatial uncertainty. Journal of the Optical Society of America, 1974, 64, 1715-1719.

Eriksen, C. W., \& Spencer. T. Rate of information processing in visual perception: Some results and methodological considerations. Journal of Experimental Psychology, Monograph, 1969, 79 (2, Pt. 2), 1-16.

GARNER, W. R. The processing of information and structure. Potomac, Md: Erlbaum, 1974.

GiBson, J. J. The ecological approach to visual perception. Boston: Houghton-Mifflin, 1979.

Green, D. M., \& Birdsall, T. G. Detection and recognition. Psychological Review, 1978, 85, 192-206.

Green, D. M., \& Swets, J. A. Signal detection theory and psychophysics. New York: Wiley, 1966.

Green, D. M., \& Weber, D. L. Detection of temporally uncertain signals. Journal of the Acoustical Society of America, $1980,67,1304-1311$.

HEIDER, F. Social perception and phenomenal causality. Psychological Review, 1944, 51, 358-374.

Julesz, B. Foundations of cyclopean perception. Chicago: University of Chicago Press, 1971.

Julesz, B. Experiments in the visual perception of texture. Scientific American, 1975, 232, 34-43.

Julesz, B. Perceptual limits of texture discrimination and their implications to figure/ground separation. In E. L. J. Leeuwenberg \& H. F. J. M. Buffart (Eds.), Formal theories of visual perception. New York: Wiley, 1978.

Julesz, B., \& BurT, P. Cooperativity of nearby micropatterns in texture discrimination. Bulletin of the Psychonomic Society, 1979, 14, 242. (Abstract)

Kinchla, R. A. Detecting target elements in multielement arrays: A confusability model. Perception \& Psychophysics, 1974, 15, 149-158.

KinCHLA, R. A. The role of structural redundancy in the perception of visual targets. Perception \& Psychophysics, 1977, 22, 19-30.

LAPPIN, J. S. The relativity of choice behavior and the effect of prior knowledge on the speed and accuracy of recognition. In N. J. Castellan, Jr., \& F. Restle (Eds.), Cognitive theory (Vol. 3). Hillsdale, N.J: Erlbaum, 1978.

LAPPIN, J. S. The relativity of perception, choice, and social knowledge. In J. H. Harvey (Ed.), Cognition, social behavior and the environment. Hillsdale, N.J: Erlbaum, in press.

Lappin, J. S., \& BeLl, H. H. The detection of coherence in moving random-dot patterns. Vision Research, 1976, 16, 161-168.

Lappin, J. S., Doner, J. F., \& Kottas, B. L. Minimal conditions for the visual detection of three-dimensional structure and motion. Science, 1980, 209, 717-719.

LAPPIN, J. S., \& UTTAL, W. R. Does prior knowledge facilitate the detection of visual targets in random noise? Perception \& Psychophysics, 1976, 20, 367-374.

LEWIN, K. Principles of topological psychology. New York: McGraw-Hill, 1936.

LuCE, R. D. Individual choice behavior. New York: Wiley, 1959.

LuCE, R. D. Detection and recognition. In R. D. Luce, R. R. Bush, \& E. Galanter (Eds.), Handbook of mathematical psychology. New York: Wiley, 1963.

Nolte, L. W., \& JAARsma, D. More on the detection of one of $M$ orthogonal signals. Journal of the Acoustical Society of America, 1967, 41, 497-505.

Peterson, W., Birdsall, T., \& Fox, W. The theory of signal detectability. IRE Transactions on Information Theory, 1954, 4, 171-212.

Pollack, I. Discrimination of third-order Markov constraints within visual displays. Perception \& Psychophysics, 1973, 13, 276-280.

Sekuler, R., \& Ball, K. Mental set alters visibility of moving targets. Science, 1977, 198, 60-62. 
Shaw, M. L. Attending to multiple sources of information. Cognitive Psychology, in press.

Shaw, R., \& Turvey, M. T. Coalitions as models for ecosystems: A realist perspective on perceptual organization. In M. Kubovy \& J. Pomerantz (Eds.), Perceptual organization. Hillsdale, N.J: Erlbaum, in press.

SHIPLEY, E. F. A model for detection and recognition with signal uncertainty. Psychometrika, 1960, 25, 273-289.

Staller, J. D., \& LAPPIN, J. S. Observers detect higher order relations in visual search for letters. Bulletin of the Psychonomic Society, 1979, 14, 258-259. (Abstract)

STALler, J. D., \& LAPPIN, J. S. Visual detection of multiletter patterns. Journal of Experimental Psychology: Human Perception and Performance, in press.

Staller, J. D., Lappin, J. S., \& Fox, R. Stimulus uncertainty does not impair stereopsis. Perception \& Psychophysics, 1980, 27, 361-367.

UtTAL, W. R. An autocorrelation theory of form detection. Hillsdale, N.J: Erlbaum, 1975.

YELLOTT, J. I. The relationship between Luce's choice axiom, Thurstone's theory of comparative judgment and the double exponential distribution. Journal of Mathematical Psychology, 1977, 15, 109-144.

\section{NOTES}

1. We use the term "coherence" to refer to the cross-correlation of the two successive patterns. This is consistent with the use of the term in statistical communications theory when referring to constraints on the distribution of events produced by some otherwise random process. This use is also consistent with the use of the term in optics and holography, in which the coherence of light may be measured by an autocorrelogram of the space-time distribution of the light amplitudes.

2. Although it is not generally recognized that stimuli cannot be defined independently of the observer, the idea is not new. A similar idea was expressed in the Gestalt approach to social cognition (Heider, 1944; Lewin, 1936), as well as by researchers applying information theory to psychology (see Lappin, in press, for a review). As Garner (1974) aptly puts it, "information is a function not of what the stimulus is, but rather of what it might have been, of its alternatives" (p. 194). From an ecological perspective, Gibson (1979) and Shaw and Turvey (in press) have pointed out that the "stimulus" is a relational concept defined by a coalition between organism and environment as coordinated parts of a dynamic system. Thus, the effective physical properties of a given stimulus event are defined in part by the observer's prior knowledge of the potential stimuli.

3. A comprehensive test and evaluation of these or alternative models is outside the scope of this paper. Our purpose is not to test these models, but to provide standards of reference with which to evaluate the obtained data. These models were chosen not because they are the best models, but because they are diagnostic in the interpretation of our experiments. We are more interested in the class of linear independence models than in these particular two models. The Peterson, Birdsall, and Fox (1954) formula is useful as an index of the upper bound on the performance expected under increased stimulus uncertainty. The choice-theory model is of interest because it has not often been applied to this problem, is mathematically elegant, computationally simple, psychologically plausible for many situations, and empirically consistent with several sets of published data.

4. The rationale for this prediction can be based on a conception of the underlying visual process as a cross-correlation of the two successive displays, as discussed by Lappin and Bell (1976). A similar rationale can also be based on a choice-theory model, in which the ratio of the probabilities of perceiving motion in any two alternative locations (or directions) is proportional to the ratio of the numbers of elements displayed in those locations. In either case, the critical assumption is that the underlying events are perceptually independent.

5. Two of the observers served for an additional set of sessions. These were conducted several weeks after the other data had been collected. The procedures were also slightly different in these additional sessions, consisting of two blocks of 200 trials rather than four blocks of 100 trials as in the rest of the experiment. We have treated these additional data as if they were produced by two additional observers, although actually only four (rather than six) different persons served as observers.

6. Kinchla (1974) also provided an excellent quantitative account of these data with his weighted integration model. This is an alternative linear model that assumes responses are based on an integration of independent sensory information about all of the stimulus alternatives. However, his integration model contained an additional free parameter corresponding to the influence of other sources of variance; without this extra parameter, the predictions of the integration model would have been close to those for the choice-theory model (although the effect of uncertainty is greater for the integration model than for the choice-theory model at higher performance levels). Obviously, Kinchla's two-parameter model could not be tested by the present data with only 2 degrees of freedom. The one-parameter version of Kinchla's model would have predicted a larger effect of uncertainty than was obtained in the present experiments.

\section{APPENDIX}

The computational formula for predicting detection accuracy as a function of the number of stimulus alternatives was a variation on the choice-theory formula used by Lappin and Uttal (1976). The basic assumptions in this formula are: (1) The observer selects the response associated with the stimulus with maximum value (e.g., maximum energy or maximum likelihood ratio). (2) All of the potential nontarget stimulus alternatives have an equal and independent chance of being confused with the true target. That is, the noise processes producing confusions between target and nontarget are assumed to be identically distributed independent random variables. (3) Finally, detection probabilities are assumed to be governed by a version of Luce's (1959) choice axiom, which may be written as follows. Let $\mathrm{P}^{*}$ be the probability of correctly identifying the target in a twoalternative forced-choice experiment (with one potentially confusable nontarget alternative), and let $P_{n}$ be the probability of correctly identifying the target within a set of $n$ alternative stimuli (with $n-1$ potentially confusable nontarget alternatives). Then we assume that

$$
\mathbf{P}_{\mathrm{n}}=\frac{\mathrm{P}^{*}}{\mathrm{P}^{*}+(\mathrm{n}-1)\left(1-\mathrm{P}^{*}\right)} \text {. }
$$

That is, the probability of a correct identification is construed as a ratio of scale values for target and nontarget alternatives, where $\mathrm{P}^{*}$ is the scale value for the target and $1-\mathrm{P}^{*}$ is the scale value for a nontarget. The probability of correctly identifying the target is assumed to depend only on the parameter $\mathrm{P}^{*}$ and on the number of nontarget alternatives.

In a forced-choice detection task, such as employed in the present experiments, the observer may sometimes choose the correct response on the basis of erroneous information, when one of the nontarget events associated with the correct response is mistaken for the true target. In a $\mathbf{k}$-alternative 
forced-choice detection task with $\mathrm{n}$ alternative stimulus events in each of the $\mathbf{k}$ subjects and the target appearing only once in one of the subsets, the total number of stimulus events is $\mathrm{kn}$, the total number of nontargets is $\mathrm{kn}-1$, and the number of nontarget alternatives associated with the correct response is $n-1$. By the same reasoning as is embodied in Equation 1, it is readily seen that

$$
P_{k, n}=\frac{P^{*}+(n-1)\left(1-P^{*}\right)}{P^{*}+(k n-1)\left(1-P^{*}\right)}
$$

where $\mathbf{P}_{k, n}$ is the probability of a correct response in a $\mathbf{k}$ alternative forced-choice task with $\mathrm{n}$ potential alternative target stimuli in each of the $\mathbf{k}$ subsets of stimuli. This is a generalization of the formula given by Lappin and Uttal (1976) for a two-alternative forced-choice task.

The rationale for this model is essentially the same as for Luce's choice theory (see Luce, 1963). This was pointed out to us by Green (Note 1), who noted that Equation 1 (as given in Lappin \& Uttal, 1976) is equivalent to Equation 27, p. 140, in Luce (1963). This model for the effects of stimulus uncertainty is also essentially the same as that developed by Luce (1963, pp. 182-183) and Shipley (1960), although the notation is somewhat different.

Although Equations 1 and 2 are not stated in terms of probability distributions of random variables associated with the target and nontarget stimulus alternatives, they do implicitly assume that the cumulative distribution function of these random variables would have a certain formspecifically, double exponential: $F(x)=\exp [-\exp (-a x+b)]$, where $a>0$ and $b$ is arbitrary (see Yellott, 1977). When choices are assumed to be made by selecting the maximum from a set of independent and identically distributed random variables (identical up to translation along the $\mathbf{P}^{*}$ axis, corresponding to the parameter b), as in Thurstonian discrimination models, then the only probability distribution that will satisfy Equation 1 for $n \geqslant 3$ is the double exponential (Yellott, 1977). Moreover, if a distribution function is the double exponential, then the corresponding Thurstonian model is equivalent to the choice axiom.

In addition to its correspondence with the choice axiom, Yellott (1977) has pointed out that the double exponential distribution possesses two other interesting properties: First, it is an asymptotic (as $\mathbf{n} \rightarrow \infty$ ) distribution of the maximum of a set of $\mathbf{n}$ independent and identically distributed random variables. Thus, the double exponential and the choice axiom are associated with the response rule of choosing the maximum. Second, the double exponential is implied by a Thurstonian discrimination model in which choice probabilities are "invariant under uniform expansion of the choice set"' (Yellott, 1977). In the present experiments, this latter property corresponds to the property that the functional relation between detection accuracy and number of stimulus alternatives is invariant under uniform expansions of the number of stimulus alternatives. That is, if we wish to predict how detection accuracy would be changed by increasing the number of directions of motion from one to four alternative directions, then the prediction is the same whether we consider each stimulus as composed of $1,10,100$, or 500 independent component motions. If Equation 2 is used to predict the performance in a condition with $\mathrm{n}$ stimulus alternatives from a condition with $m$ alternatives, then the prediction depends only on the ratio $\mathrm{n} / \mathrm{m}$ rather than on the absolute values of $n$ and $m$. Clearly, this invariance property is useful in the present experiments where there is ambiguity about the number of perceptual components in these dynamic random-dot patterns. It turns out that the Peterson, Birdsall, and Fox (1954) formula for the effect of stimulus uncertainty also possesses a corresponding invariance under uniform expansions of the number of stimulus alternatives, but the response rule in this case is based on averaging likelihood ratios over all the stimulus alternatives associated with a given response rather than on the maximum likelihood ratio. (That is, the Peterson et al. model is not a Thurstonian model in this situation.)

Since it is often informative to represent detectability as a discriminal distance between stimuli, and since the choicetheory measure $-\log \eta$ provides a convenient and meaningful example of such a measure, it is useful to transform Equation 2 into an expression in terms of the measure $-\log \eta$. This measure is similar to the measure $d^{\prime}$ in signal detection theory when the noise and signal-plus-noise distributions are assumed to have equal variance. The choice theory implicitly assumes that these distributions are double exponential, and double exponential distributions are not discriminably different from normal distributions in most empirical detection experiments. The choice-theory measure offers advantages in ease of computation and, more importantly for present purposes, a means of obtaining exact predictions for the relation between the detectability measure $-\log \eta$ and the number of stimulus alternatives, $\mathrm{n}$. An exact formulation of this relationship for the ideal observer cannot be obtained from signal detection theory with normal distributions.

To transform Equation 2 into an expression in $-\log \eta$, first note that the relation between the parameters $P^{*}$ and $\eta$ (as defined by Luce, 1963) is

$$
\begin{gathered}
\mathrm{P}^{*}=\frac{1}{1+\eta^{\sqrt{2}}} \\
\eta^{\sqrt{2}}=\frac{1-\mathrm{P}^{*}}{\mathrm{P}^{*}}
\end{gathered}
$$

(The exponent, $\sqrt{2}$, derives from the fact that $P^{*}$ is defined by a two-alternative forced-choice detection experiment with two independent observations on each trial. See Luce, 1963, pp. 139-140.) In a k-alternative forced-choice detection experiment, where $P_{k}$ is the observed percentage of correct responses, we have

$$
\eta^{\sqrt{2}}=\frac{1-P_{k}}{(k-1) P_{k}}
$$

By substituting Equation 3 into Equation 2, we can write

$$
P_{k, n}=\frac{1+(n-1) \eta^{\sqrt{2}}}{1+(k n-1) \eta^{\sqrt{2}}}
$$

and

$$
\frac{1-P_{k, n}}{(k-1) P_{k, n}}=\frac{n \eta^{\sqrt{2}}}{1+(n-1) \eta \sqrt{2}} .
$$


Let us now denote the detectability measure $-\log \eta$ as $D$, and denote the predicted detectability for $n$ stimulus alternatives as $D_{n}$. By definition,

$$
\eta \equiv e^{-D} \text { and } \eta^{\sqrt{2}} \equiv e^{-\sqrt{2}} \mathrm{D}
$$

From Equations 4 and 6, we can write

$$
\begin{aligned}
D_{n} & =-(1 / \sqrt{2}) \log \left[\frac{n \eta^{\sqrt{2}}}{1+(n-1) \eta^{\sqrt{2}}}\right] \\
& =-(1 / \sqrt{2}) \log \left[\frac{n \exp (-\sqrt{2} D)}{1+(n-1) \exp (-\sqrt{2} D)}\right] \\
& =(1 / \sqrt{2}) \log \left[\frac{\exp (\sqrt{2} D)+(n-1)}{n}\right] .
\end{aligned}
$$

Equation 7 gives the predicted relation between detectability and the number of stimulus alternatives. This equa- tion is isomorphic with Equation 2 expressed in terms of the observed percentage of correct responses.

Equation 7 is similar to a relationship that can be derived from signal detection theory by assuming orthogonal noise distributions that are normal with equal variance. An exact formulation of the latter relationship cannot be given, however, because an algebraic expression cannot be written for the distribution of likelihood ratios in this situation. Peterson, Birdsall, and Fox (1954) give a formulation based on an approximation to the likelihood ratio distribution, and Nolte and Jaarsma (1967) present empirical functions obtained by numerical iteration. Green and Birdsall (1978) and Nolte and Jaarsma (1967) describe a close approximation to these empirical functions provided by a set of independent threshold detectors.

\footnotetext{
(Received for publication March , 1980; revision accepted January 14,1981 )
} 